usual setting (hospital or community) to another (the hospice) to learn.

Methods Qualitative semi-structured interviews were conducted with ten purposively recruited healthcare professionals who were visiting Meadow House Hospice for experiential learning. Data analysis involved a framework approach to recognise themes confirming the visitors as educational tourists. Interview data was then used to generate themes to inform an educational programme.

Results Four student nurses, 2 medical students, a district nurse, a GP trainee, a hospital specialist breast nurse and an advanced medical practitioner visiting the hospice showed characteristics common to educational tourists: travelling to learn, a desire to learn, wishing to experience something different, wishing to experience something in context and a preference for experiential learning. Visitors considered their experience to be beneficial to their future practice and three main themes about how to improve the hospice educational programme were established; personalising learning; continuing to see and experience authentic hospice activities; and the presence of a facilitator guide.

Conclusion This study confirms that educational tourism occurs in the hospice, substantiating the application of educational tourism models. Using the pre-visit, visit, post-visit educational tourism model, improvements could include: pre-visit personalisation and planning of the learning experience by clarifying objectives, giving information and establishing visitor preferences about educational activities; postvisit debriefing/reflection and outcome measurement while maintaining the current authentic learning experience with a facilitator guide. Using an educational tourism model may offer an opportunity to improve learning experiences in the hospice within the educational resources already available.

\section{MEETING THE EDUCATIONAL OBJECTIVES OF HEALTHCARE PROFESSIONAL VISITORS TO MEADOW HOUSE HOSPICE}

Treena Saini. London North West University Healthcare NHS Trust

10.1136/spcare-2020-PCC.59

Introduction Meadow House Hospice provides experiential learning to healthcare professionals who request it. A review was undertaken to understand learners' objectives and whether these could be met in a short visit.

Methods Visitors were invited to fill in a questionnaire pre and post visit to identify educational objectives. This information was used to conduct a retrospective thematic analysis.

Results From April 2017 to April 2019, the hospice hosted 60 visitors (32 nurses in training, 11 doctors in training, 9 nurses, 4 doctors and 3 allied health professionals) and 206 educational objectives were documented.

Thematic analysis characterised objectives into four main themes. These were:

- visitors wanting to be more knowledgeable about the service $(43 \%)$ in particular roles and service provision (21\%), referral processes $(19 \%)$ and the hospice service in relation to their own role $(3 \%)$

- visitors wanting to gain specialist knowledge and skills (31\%) about assessment and management (22\%), medication used in end of life care patients (6\%) and communication skills (3\%)
- visitors wanting to be more knowledgeable about palliative care/specialist palliative care/end of life care (18\%)

- visitors wanting to experience the specialist palliative care service from a patient or professional perspective by attending multidisciplinary and referral meetings (8\%).

When asked how these objectives were achieved during their visit, all visitors cited one, a combination or all of the following activities: shadowing a community nurse or ward doctor; observing multidisciplinary teams and referral meetings where real time patient discussions occurred; and by interacting with hospice professionals.

Conclusion Visitors do not just seek highly specialised knowledge but want to become more knowledgeable about hospices and how they work from their own perspective. Identifying and understanding learning objectives helps meet learning needs of a wide range of healthcare professionals within a short visit to the hospice.

\section{9 'SHEDDING LIGHT ON THE SHADOWS: AN EVALUATION OF THE EXPERIENCE OF SHADOWING THE HOSPITAL PALLIATIVE CARE TEAM}

Suzanne Ford-Dunn, Kirsty Sayers. Western Sussex Hospitals NHS Foundation Trust, St Barnabas House Worthing

\subsection{6/spcare-2020-PCC.60}

Background The hospital palliative care team (HPCT) regularly welcomes healthcare professionals (HCP) to spend time 'shadowing them - observing the team in practice and receiving informal 1 to 1 teaching. There is little data available on the benefits of this type of experience. We aimed to evaluate the benefits through analysis of the visiting HCP feedback forms.

Aim To understand the learning experience and benefits of a shadowing placement with the HPCT.

Methods Evaluation forms were collected over a 2 year period. The content was grouped and analysed using a basic thematic analysis.

Results There were 52 completed evaluation forms. These 52 individuals spent a total of 93 days shadowing the team, the average length of shadow experience was 1.8 days. The HCP were predominantly nursing or medical students (24 and 8 respectively). The remainder were a broad multi professional spread: occupational therapy, cancer nurses, discharge coordinator, H@H nurses etc. Of the 52 HCP, 50 described themselves as 'very satisfied' with the placement and 51 found it very useful . Analysis of free text revealed 3 major and 4 minor themes. Major themes were Understanding general principles of palliative care (PC), Understanding the role of the HPCT, and Inspiring professional growth. Minor themes included PC drugs/prescribing, Observing good communication, Providing learning resources, and Multidisciplinary team working.

Conclusion The participants overwhelmingly described the experience as useful, with expected benefits in terms of developing general PC knowledge and understanding the role of the HPCT. Unexpected, was the theme of Inspiring professional development, with many HCP commenting that they felt inspired to study more, to think about a career in palliative medicine or just more inspired about their own role in healthcare. This suggests that the benefits of this type of placement extend beyond the individual, to the wider healthcare team and employing organisation. 